\title{
Primeiro registro de Aedes albopictus (Diptera: Culicidae) em Roraima, Brasil
}

\author{
Ducineia Barros AGUIAR ${ }^{1}$, Almir FONTÃO², Pedro RUFINO³, Valdenor Alves MACEDO ${ }^{4}$, Cláudia Maria \\ RÍOS-VELÁSQUEZ ${ }^{5}$, Márcia Gonçalves $\mathrm{CASTRO}^{6}$, Nildimar Alves $\mathrm{HONÓRIO}^{7}$ \\ RESUMO \\ Aedes albopictus é registrado pela primeira vez no estado de Roraima, Brasil. Entre junho de 2006 e maio de 2007 foram \\ coletadas três pupas e dez larvas, duas das quais chegaram à fase adulta, durante atividades de vigilância rotineiras em três bairros \\ urbanos da cidade de Boa Vista. Embora essa espécie não seja incriminada como vetor primário do dengue, a sua presença \\ pode favorecer a ligação entre os ciclos silvestre e urbano da febre amarela e de outras arboviroses no Brasil.
}

PALAVRAS-CHAVE: Aedes albopictus, Dengue, Roraima

\section{First record of Aedes albopictus (Diptera: Culicidae) in the state of Roraima, Brazil}

\section{ABSTRACT}

Aedes albopictus is registered for the first time in Roraima, Brazil. From June 2006 to May 2007, three pupae and ten larvae of Ae. albopictus were collected, during routine surveillance work in three urban neighborhoods in the city of Boa Vista. Two larvae reached adulthood as females. Although Ae. albopictus is not presently considered of primary importance in dengue transmission, its occurrence could favor a linkage between urban and forest cycles of yellow fever and other arboviruses in Brazil.

KEYWORDS: Aedes albopictus, Dengue, Roraima

1 Bióloga do Núcleo Estadual de Entomologia, Secretaria de Estado da Saúde de Roraima e-mail: ducibio10@yahoo.com.br.

2 Agente de Endemias da Prefeitura Municipal de Saúde de Boa Vista e do Núcleo Avançado de Vetores da Universidade Federal de Roraima.e-mail: afc@click21.com.br

${ }^{3}$ Agente de Saúde Pública, Núcleo Estadual de Entomologia, Secretaria de Estado da Saúde de Roraima email: pedrorufino@uol.com.br.

${ }^{4}$ Agente de Saúde Pública, Núcleo Municipal de Entomologia, Secretaria de Saúde de Boa Vista, Roraima e-mail: valdenoralves@yahoo.com.br.

5 Pesquisadora do Laboratório de Biodiversidade em Saúde do Centro de Pesquisa Leônidas \& Maria Deane - FIOCRUZ/AM. e-mail: crios@amazonia.fiocruz.br

${ }_{6}^{6}$ Pesquisadora do Laboratório de Transmissores de Hematozoários, Departamento de Entomologia do Instituto Oswaldo Cruz - FIOCUZ. e-mail: mcastro@ioc.fiocruz.br

7 Pesquisadora do Laboratório de Transmissores de Hematozoários, Departamento de Entomologia do Instituto 0swaldo Cruz - FIOCUZ. e-mail: honorio@ioc.fiocruz.br 
Aedes albopictus (Skuse 1894) é uma espécie oriunda do sudeste da Ásia, onde é considerado o vetor primário do vírus dengue (Hawley, 1988). No Brasil, foi registrado primeiramente em 1986 no Rio de Janeiro e em Minas Gerais. Posteriormente, foi invadindo estados vizinhos, como São Paulo e Espírito Santo (Consoli \& Lourenço-de-Oliveira, 1994). Até a presente comunicação, apenas 6 dos 27 estados brasileiros ainda não registraram a ocorrência dessa espécie: Amapá, Acre, Tocantins, Piauí, Sergipe e Roraima (Santos 2003; Martins et al., 2006).

A presença de Ae. albopictus é um sério problema para a saúde pública. Apesar de Ae. albopictus ainda não ter sido incriminado como vetor natural do dengue no Brasil (Schatzmayr, 2000), foi comprovado que em condiçōes de laboratório e de campo, populações brasileiras desta espécie têm a capacidade de se infectar com o vírus do dengue e transmiti-lo (Lourenço-de-Oliveira et al., 2003; Castro et al., 2004).

Ao contrário do Ae aegypti, Ae. albopictus se espalha nos ambientes urbano, suburbano e rural, não dependendo de locais de grande concentração humana (Consoli \& Lourençode-Oliveira, 1994). Além disso, apresenta ampla plasticidade ecológica evidenciada pela capacidade de colonizar os mais variados tipos de recipientes, naturais e artificiais (Hawley, 1988). O presente estudo teve o objetivo de registrar a primeira ocorrência de Ae. albopictus no estado de Roraima, Brasil.

Os primeiros exemplares de Ae. albopictus foram coletados durante as atividades de rotina do Programa de Vigilância e Controle do Dengue na cidade de Boa Vista. Agentes de endemias realizam visitas domiciliares bimestralmente em busca de criadouros contendo formas imaturas do Aedes aegypti, além de implantarem armadilhas de oviposição em cinco bairros da cidade para obtenção dos índices de infestação. Durante uma dessas visitas, em junho de 2006, foram coletadas duas pupas de Ae. albopictus (1 macho e 1 fêmea) no bairro Araceli Souto Maior (02 46 44.4” N; $60^{\circ}$ $43^{\prime} 10.8^{\prime}$ W), em um depósito abandonado no quintal de uma residência. Em novembro de 2006 foi coletada mais uma pupa fêmea de Ae. albopictus, em um depósito artificial no peridomicílio de uma residência localizada no bairro Cinturão Verde ( $02^{\circ} 48^{\prime} 00.7^{\prime \prime} \mathrm{N}$; 60 41' 56.9” W), que fica a $50 \mathrm{~m}$ do Igarapé Pricumã. Em maio de 2007, foram coletadas 10 larvas de Ae. albopictus, em uma ovitrampa, localizada no bairro Pricumã $\left(02^{\circ} 48^{\prime} 21,9^{\prime \prime} \mathrm{N}\right.$; $60^{\circ} 41^{\prime} 50,5^{\prime \prime}$ W), situado na zona oeste da cidade de Boa Vista. Destas, apenas 2 chegaram à fase adulta. Os bairros onde as 3 pupas e 10 larvas de Ae. albopictus foram coletadas apresentam ampla cobertura vegetal, acompanhados por extensa mata aluvial e cortados pelos Igarapés Uai Grande e Pricumã (Figura 1). As larvas, as pupas e os adultos do mosquito coletados nesse trabalho foram identificados seguindo a chave dicotômica

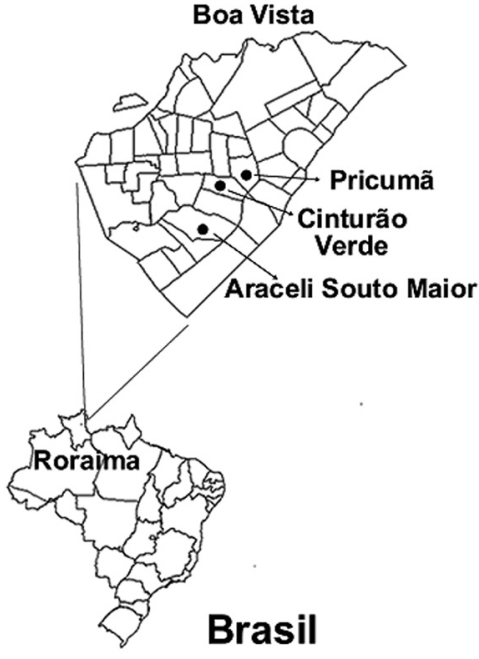

Figura 1 - Mapa da ocorrência de Aedes albopictus nos bairros Pricumã, Cinturão Verde e Araceli Souto Maior, Boa Vista, Roraima.

de Consoli \& Lourenço-de-Oliveira (994). As larvas e pupas foram mantidas em álcool a 70\% e os adultos montados em alfinetes entomológicos. Os espécimes foram depositados na coleção entomológica do Núcleo Avançado de Vetores do Departamento de Medicina da Universidade Federal de Roraima - UFRR.

O estado de Roraima é considerado uma porta de entrada de patógenos em decorrência da sua posição geográfica (acesso ao Brasil a partir do Caribe, Venezuela e América Central), características climáticas e alta incidência de dengue (Rosa-Freitas et al., 2003). A rodovia BR-174, que liga Manaus à Caracas, proporciona um intenso fluxo de pessoas vindas da região do Caribe (onde a dengue é endêmica) e de Manaus (onde doenças como a dengue e a febre amarela são endêmicas), tornando as cidades que margeiam a rodovia, como é o caso de Boa Vista, particularmente suscetíveis à epidemias. Além disso, foi em Roraima que houve o primeiro registro clínico e laboratorial de dengue em 1981-1982 (Osanai et al., 1983).

Embora o Ae. albopictus seja considerado vetor potencial de dengue (Schatzmayr, 2000), seus aspectos ecológicos tais como: eclético quanto a alimentação sangüínea (Consoli \& Lourenço-de-Oliveira, 1994), capacidade de dispersão (Honório et al., 2003) segregação espacial, competição interespecífica (Braks et al., 2003, 2004) e adaptabilidade ecológica, podem favorecer a ligação entre os ciclos silvestre e urbano da febre amarela e de outras arboviroses no Brasil (Braks et al., 2003, Lourenço-de-Oliveira et al., 2003). 


\section{AGRADECIMENTOS}

Os autores agradecem aos agentes de endemias Davi Ribas Galvão, Hailton Mariano, Rosângela Santos, Francisco Maciel, Gerson Souza, Valdiney Gomes e Rodrigo de Oliveira Brasil, pela assistência nos trabalhos de campo e laboratório e a Secretaria de Vigilância em Saúde - SVS, pelo suporte financeiro.

\section{BIBLIOGRAFIA CITADA}

Braks, M.A.; Honório, N.A.; Lounibos, L.P.; Lourenço-de-Oliveira, R.; Juliano, S.A.; 2004. Interspecific competition between two invasive species of container mosquitoes in Brazil. Ann. Entomol. Soc. Am., 97: 130-139.

Braks, M.A.; Honório, N.A.; Lourenço-de-Oliveira, R.; Juliano, S.A.; Lounibos, L.P. 2003. Convergent habitat segregation of Aedes aegypti and Aedes albopictus (Diptera: Culicidae) in southeastern Brazil and Florida. J. Med. Entomol., 40: 785-794.

Castro, M.G.; Nogueira, R.M.R.; Schatzmayr, H.G.; Miagostovich, M.P.; Lourenço-de-Oliveira, R. 2004. Dengue virus detection by using reverse transcription-polymerase chain reaction in saliva and progeny of experimentally infected Aedes albopictus from Brazil. Mem Inst Oswaldo Cruz, 99: 809-814.

Consoli, R.A.G.B; Lourenço-de-Oliveira, R. 1994. Principais mosquitos de importância sanitária no Brasil, Fiocruz, Rio de Janeiro. 225pp.

Hawley, W.A. 1988. The biology of Aedes albopictus. J. Am. Mosq. Control Assoc., 1: 1-39.
Honório, N.A.; Silva, W.C.; Leite, P.J.; Gonçalves, J.M.; Lounibos, L.P.; Lourenço-de-Oliveira, R. 2003. Dispersal of Aedes aegypti and Aedes albopictus (Diptera: Culicidae) in an urban endemic dengue área in the state of Rio de Janeiro, Brazil. Mem Inst Oswaldo Cruz, 98: 191-198.

Lourenço-de-Oliveira, R.; Vazeille, M.; Filippis, A.M.B.; Failloux, A.B. 2003. Aedes albopictus from Brazil and southern United States: genetic variation and vector competence for dengue and yellow fever viruses. Am J Trop Med Hyg 69: 105-114.

Martins, V.E.P.; Martins, M.G.; Araújo, J.M.P.; Silva, L.O.R; Monteiro, H.A.O.; Castro, F.C.; Vasconcelos, P.F.C.; Guedes, M.I.F. 2006. Primeiro registro de Aedes (Stegomyia) albopictus no Estado do Ceará, Brasil. Rev Saúde Pública, 40(4): 737-9.

Osanai, C.H.; Travassos-da-Rosa, A.P.A.; Tang, A.T.; Amaral, R.S.; Passos, A.D.C.; Tauil, P.I. 1983. Surto de dengue em Boa Vista, Roraima. Rev Inst Med Trop São Paulo, 25(4): 53-54.

Rosa-Freitas, M.G.; Tsouris, P.; Sibajev, A.; Weimann, E.T.S.; Marques, A.U.; Ferreira, R.L.; Luitgards-Moura, J.F. 2003. Exploratory Temporal and Spatial Distribution Analysis of Dengue Notifications in Boa Vista, Roraima, Brazilian Amazon, 1999 to 2001. Dengue Bulletin, 27: 63-80.

Santos, R.C. 2003. Updating of the distribution of Aedes albopictus in Brazil (1997 - 2002). Rev Saúde Pública, 37(5): 1-4.

Schatzmayr, H.G. 2000. Dengue situation in Brazil by year 2000. Mem Inst Oswaldo Cruz, 95(Supl 1): 179-181.

Recebido em 07/08/2007

Aceito em 26/03/2008 
